Para enlazar con este artículo / To link to this article:

http://dx.doi.org/10.14198/fem.2017.29.13

Para citar este artículo / To cite this article:

Rausell Guillot, Helena y Talavera Ortega, Marta. «Dificultades de la coeducación en la formación del profesorado». En Marcos Jesús Iglesias Martínez e Inés Lozano Cabezas (coords.), La (in)visibilidad de las mujeres en la Educación Superior: retos y desafíos en la Academia. Feminismo/s, 29 (junio 2017): 329-345, DOI: 10.14198/fem.2017.29.13

\title{
DIFICULTADES DE LA COEDUCACIÓN EN LA FORMACIÓN DEL PROFESORADO ${ }^{1}$
}

\section{DIFFICULTIES IN COEDUCATION IN TEACHING TRAINING}

\author{
Helena RAUSELL GUILLOT \\ helena.rausell@uv.es \\ orcid.org/0000-0003-1803-3487
}

Marta TALAVERA ORTEGA

marta.talavera@uv.es orcid.org/0000-0002-1250-0200

Universitat de València

\section{Resumen}

La situación de la implementación del género y la diversidad sexual en las etapas de Educación Infantil y Primaria es heterogénea en el conjunto de España. El presente estudio se centra en analizar la situación de la perspectiva de género en el Máster de Investigación en Didácticas Específicas de la Universitat de València, como parte de la formación especializada que se oferta desde la Facultad de Magisterio para futuros docentes y otros formadores. También trata de hacer una propuesta metodológica para contribuir a la formación especializada en investigación desde la perspectiva de género.

Palabras clave: coeducación, género, igualdad, formación inicial del profesorado, didáctica.

1. Este trabajo ha contado con financiación del Plan Nacional de I+D+i del Gobierno de España (EDU2015-65621-C3-1-R ) y cofinanciado con fondos FEDER de la Unión Europea.

Aquest treball ha estat realitzat en el marc del programa de Doctorat en Educació de la Universitat Autònoma de Barcelona. 


\begin{abstract}
Introducing gender perspective and sexual diversity in primary and early school education is heterogeneous right now in Spain. Our study focuses on gender implementation in the Master of Specific Didactics in the University of Valencia, as a part of the specialized teaching and researching training which is offered by the Teaching Training Faculty. The article also tries to design a methodological proposal in order to contribute to a specialized researching training from gender perspective.
\end{abstract}

Keywords: coeducation, gender, equality, teacher training, didactics. 


\section{APROXIMACIÓN TEÓRICA}

La coeducación se entiende como un proceso educativo intencionado que tiene el objetivo de desarrollar de forma integral a las personas, pero que parte de la consideración de que éstas tienen una realidad diferenciada según su sexo. El término se ha venido aplicando tradicionalmente a la educación en compañía de alumnos y alumnas en un mismo centro escolar, aunque coeducar puede también entenderse como educar fuera del modelo dominante, el simbólico patriarcal, pensando de nuevo, desde otros referentes, qué significa ser una mujer o ser un hombre, en el contexto histórico en el que vivimos (Blanco 20). Nos situaríamos, de este modo, en un proceso en el que se trataríamos de formar a estas personas para una convivencia social igualitaria, para lo cual la escuela mixta es un necesario punto de partida (Heredero de Pedro 139) y la implementación de estudios de género, una necesidad.

Sin embargo, la situación de la implementación del género y la diversidad sexual en las etapas de Educación Infantil y Primaria es heterogénea en el conjunto de España, a pesar de que, en algunas comunidades autónomas, en los últimos años, se ha dado un cierto impulso a las temáticas de género. Es el caso de Andalucía y, recientemente, también de Cataluña, donde, por ejemplo, en los centros es obligatoria la existencia de un responsable de coeducación y la inclusión de la perspectiva de género en el Plan de Desarrollo del centro (Romero 50). En la Comunidad Valenciana, recientemente, se ha publicado la Instrucción por la que se establece el protocolo de acompañamiento para garantizar el derecho a la identidad de género, la expresión de género y la intersexualidad (27 de diciembre de 2016). En otras comunidades, sin embargo, no se contempla ninguna actuación al respecto o bien se han implementado programas de coeducación que son voluntarios para los centros y que están dotados de escaso presupuesto.

Esto mismo se repite a nivel de la formación inicial del profesorado, a pesar de que estos procesos formativos son una oportunidad clave para entrenar la mirada y hacerla capaz de captar lo más fielmente posible la realidad y las necesidades de cada aula (Jaramillo 9). De hecho, un buen número de estudios recientes se ha centrado en la relevancia de las propuestas para la 
formación inicial y continua del profesorado y en la dignificación de su trabajo como personal autónomo, crítico y creativo, con capacidad de tomar decisiones sobre el por qué, el qué y el cómo enseñar (Pagès 47). También en la importancia de una adecuada selección de contenidos y materiales que contribuya a desarrollar el pensamiento crítico en el alumnado, entendiendo por tal la capacidad de problematizar lo evidente, de presentar en forma problemática a los estudiantes los contenidos, saber relacionar los hechos con valores o buscar la comprensión. Por todo ello, consideramos que a pesar de que el género está presente en algunas de las guías docentes de los grados de educación, su presencia podría y debería ser mucho más evidente y relevante en los planes de formación (Rebollar 159).

En el caso de la Universitat de València, no aparece ninguna referencia al género en el plan de estudios para Grado en Maestro/a en Educación Primaria y sólo en las competencias específicas (CE33) se hace una alusión a «conocer, comprender y respetar las distintas culturas y las diferencias entre las personas, la igualdad de derechos y oportunidades de hombres y mujeres y la no discriminación de personas con discapacidad». También podemos encontrar una mención a la coeducación (CE7) como la capacidad de «conocer la dimensión pedagógica de la interacción con los iguales y los adultos, la relacionada con la coeducación, y saber promover la participación en actividades colectivas, el trabajo cooperativo y el esfuerzo individual.»

Por tanto, en lo tocante a las propuestas y cuestionamientos que son planteados a los docentes durante su formación, así como en lo que respecta a las conclusiones del análisis de las políticas de igualdad nos encontramos en un momento crucial para su avance en las universidades, un momento en el que el objetivo debiera ser no sólo la mejora en la actuación por la igualdad, sino también una oportunidad para que la Universidad se convirtiese en líder y modelo para la sociedad en la lucha por la igualdad (Elizondo 44)

Un gran obstáculo a la implementación de estas temáticas es la falta de materiales y métodos. A pesar de que en los últimos años han aparecido cuentos, materiales didácticos y audiovisuales, éstos aún no son suficientes, tal como indican los datos analizados (Romero 51). Sin embargo, a nivel legislativo se ha dado impulso a los temas de igualdad de género. En concreto, la Ley de Igualdad aprobada en marzo de 2007 contempla en su artículo 23 diversas actuaciones educativas a favor de la igualdad de género y la introducción del mainstreaming de género en la educación. Concretamente, uno de sus apartados hace mención a la integración del estudio y aplicación del principio de igualdad en los cursos y programas para la formación inicial y permanente del 
profesorado aunque queda por determinar de qué modo se concretarán los aspectos de esta ley en los centros de formación.

Esto viene completado, a su vez, por la Ley Orgánica 8/2013, de 9 de diciembre, de mejora de la calidad educativa, que hace suya la Recomendación (2002) 12 del Comité de Ministros del Consejo de Europa a los estados miembros sobre la educación para la ciudadanía democrática, de fecha 16 de octubre de 2002, que señala que la educación para la ciudadanía democrática es esencial para promover una sociedad libre, tolerante y justa y contribuye a defender los valores y principios de la libertad, el pluralismo, los derechos humanos y el imperio de la ley, que son los fundamentos de la democracia.

Creemos que es esencial, en este sentido, que los docentes en formación entiendan que el género es un elemento clave tanto de la socialización primaria como de la socialización secundaria, que está obviamente presente ya en la primera construcción identitaria de los individuos, junto a otros elementos significativos como el lenguaje, la nacionalidad, la clase social o la religión. También que sean conscientes del papel jugado por la interacción con sujetos ajenos al entorno familiar e instituciones como la escuela, como parte de un proceso de socialización secundaria igualmente influyente.

Dentro de esta formación y dentro de la oferta de postgrado de la Facultad de Magisterio de la Universitat de València se incluye, además de otros, el Máster en Investigación en Didácticas Específicas como título oficial. Este título está enfocado a formar investigadores especializados en interrogantes didácticos relacionados con las diferentes áreas curriculares de las enseñanzas no universitarias, por ejemplo, los procesos de aprendizaje, los estilos de enseñanza, la actividad del profesorado en ejercicio, etc., igualmente en cuestiones didácticas de las enseñanzas universitarias y de cualquier otro contexto relacionado con la enseñanza y el aprendizaje de estas áreas curriculares, por ejemplo la formación inicial de profesorado, aspectos socio-culturales, elementos de historia, etc. Su objetivo último es formar investigadores que se incorporan a las comunidades investigadoras nacional e internacional de su especialidad mediante la integración en equipos existentes o la creación de nuevos equipos. Este máster está dirigido a personas licenciadas o graduadas en especialidades curriculares que tienen entre sus salidas profesionales la enseñanza en Educación Infantil, Educación Primaria o Educación Secundaria, además de estar destinada al profesorado de las áreas de conocimiento universitarias responsables de las diferentes especialidades y a aquellos que aspiren a ser profesores en estas áreas de conocimiento.

En un curso de especialización en investigación didáctica como éste creemos que es necesario poner de manifiesto el papel jugado por las mujeres en 
la investigación científica y enfatizar cómo, desde la primera manifestación por los derechos de la mujer en 1908, el número de mujeres que acceden a la Universidad ha crecido de manera continua. Sin embargo, esto no se ha acompañado de un crecimiento similar en el número de mujeres que ocupan posiciones de liderazgo en la Universidad (Menéndez 331; Rausell 37).

La razón de proponer una materia con perspectiva de género dentro del Máster de Didácticas Específicas descansa en la relevancia que consideramos que el concepto de género tiene para la investigación y la formación de los futuros docentes, pero también para una educación basada en la justicia social y el fomento de la igualdad. Se apoya, igualmente, en la creencia de que la renovación en la enseñanza de las ciencias y de todos los saberes en general pasa, necesariamente, por dar voz a otros protagonistas de las Ciencias Experimentales y Sociales y por recuperar el papel de aquellos sujetos que hasta ahora habían sido relegados al anonimato y a la invisibilidad (Valls 44; Blanco 20).

Por todo ello, desde el Departamento de Didáctica de las Ciencias Experimentales y Sociales de la Facultad de Magisterio de la Universitat de València se propone una línea de formación dentro del Máster de Investigación en Didácticas Específicas donde se trabajarían las cuestiones relacionadas con las identidades de género, la coeducación y la igualdad de manera transdisciplinar desde las áreas de conocimiento de Didáctica de las Ciencias Experimentales y Didáctica de las Ciencias Sociales.

\section{IMPORTANCIA DE LA INVESTIGACIÓN EN GÉNERO}

\subsection{Desde la perspectiva de la Didáctica de las Ciencias Experimentales}

En el caso del área de la Didáctica de las Ciencias Experimentales, el interés ha estado centrado en resaltar el papel de las mujeres en el mundo de la ciencia y su transmisión, analizando sus roles y representatividad en las diferentes disciplinas científicas, para, de este modo, conocer cómo las mujeres, una vez que consiguen entrar en el mundo universitario y hacerse un hueco en él, pueden hacer valer sus títulos e investigaciones y contribuir a la enseñanza y a la didáctica de las Ciencias Experimentales.

Por ello se pretende impulsar el desarrollo de trabajos que tengan como objetivo conocer de qué manera se pueden integrar los estudios de ciencias en los currículos académicos elementales, de manera que estos conocimientos puedan ser realmente asimilados por los alumnos y alumnas. De este modo, se pretende generar un interés por el "cómo enseñar bien», dados los retrasos existentes no sólo en la acumulación de conocimientos sino en la capacidad de discurrir y de solucionar problemas de metodologías pedagógicas que no tiene en cuenta la coeducación en las clases de ciencias. 


\subsection{Desde la perspectiva de la Didáctica de las Ciencias Sociales}

Desde el área de la Didáctica de las Ciencias Sociales se aborda la cuestión del género, en primer lugar, a partir de una reflexión sobre los usos públicos de las Ciencias Sociales y su función socio-educativa. Este tema está relacionada con el modo en el que las Ciencias Sociales participan en el proceso de socialización del individuo y aparece vinculado, más concretamente, con cómo éstas contribuyen a la construcción de las identidades en general y de las identidades de género en particular, a través de la contraposición entre identidad y alteridad, entre nosotros y ellos, entre femenino y masculino.

Para ello, creemos que podemos empezar por presentar los orígenes de la perspectiva de género en las Ciencias Sociales a partir de las críticas realizadas por el feminismo o el marxismo en los años setenta del siglo XX que señalaron la invisibilidad de las mujeres en la historia y en las restantes Ciencias Sociales. También por subrayar que una de sus principales aportaciones ha sido la necesidad de considerar la diferencia de los sexos como un asunto relevante en el análisis de las sociedades. Nuestro objetivo sería tratar de conseguir que el alumnado entendiese que las categorías de feminidad y masculinidad no son algo natural, algo dado, sino que por el contrario poseen un carácter plenamente histórico y contingente y que su significado varía de unas sociedades y culturas a otras, además de evolucionar en el tiempo. De hecho, las relaciones entre los sexos y el papel de las mujeres en cada sociedad se construyen a través de discursos y prácticas, de ordenamientos legales, instituciones, costumbres, imágenes y símbolos, formulándose en términos de diferencia o de complementariedad e incluyendo elementos de desigualdad y jerarquía y diferentes formas de inclusión y exclusión, de colaboración y dependencia (Amelang y Nash 43).

Por todo lo expuesto, el objetivo del presente trabajo sería, por una parte, poner de manifiesto la presencia creciente de las mujeres en la Universidad y, por otra, realizar una propuesta metodológica concreta para contribuir a la formación especializada en investigación dentro del marco del Máster de Investigación en didácticas específicas de la Universitat de València.

\section{METODOLOGÍA}

\subsection{Análisis de la presencia de mujeres en el Máster de Didácticas Específicas}

Para realizar el análisis de la presencia femenina en el Máster de Didácticas Específicas de la Universitat de Valencia, se estudió su plan de estudios, las competencias básicas propuestas y la composición de su equipo directivo y docente. Este examen se realizó en base a los indicadores propuestos en la Guía 
de Coeducación del Instituto de la Mujer (2011), donde se incluye una batería de indicadores para llevar a cabo el seguimiento de algunos de los aspectos que resultan claves en la igualdad de oportunidades en la educación de niños y niñas. Éstos están desarrollados como parte de sus contenidos, a modo de resúmenes breves y manejables de otros estudios y publicaciones impulsados por el Instituto de la Mujer sobre la Coeducación en España y que se valieron, a su vez, de una extensa bibliografía para su realización.

En este caso, se utilizan los indicadores para la formación del profesorado que se indican en la siguiente tabla (tabla 1).

Tabla 1. Indicadores de coeducación en la formación del profesorado

\begin{tabular}{|c|c|}
\hline Indicador & $\begin{array}{l}\text { Preguntas a responder por las } \\
\text { autoridades educativas }\end{array}$ \\
\hline $\begin{array}{l}\text { Grado de importancia otorgado por el } \\
\text { futuro profesorado a la formación en } \\
\text { IGOP }\end{array}$ & $\begin{array}{l}\text { ¿Cuál es la valoración general de los } \\
\text { futuros profesores y profesoras sobre la } \\
\text { importancia de la formación en IGOP? } \\
\text { (referente a todo el alumnado, cursen o no } \\
\text { asignaturas relativas a IGOP) }\end{array}$ \\
\hline $\begin{array}{l}\text { Proporción de tiempo asignado en } \\
\text { materias troncales a la IGOP }\end{array}$ & $\begin{array}{l}\text { Del total de créditos de asignaturas } \\
\text { troncales por especialidad y curso ¿qué } \\
\text { porcentaje representan las dedicadas a la } \\
\text { formación en IGOP? }\end{array}$ \\
\hline $\begin{array}{l}\text { Proporción de tiempo lectivo asignado a } \\
\text { asignaturas sobre IGOP, entre las propias } \\
\text { ofertadas por el centro, al margen de las } \\
\text { troncales. }\end{array}$ & $\begin{array}{l}\text { Del total de créditos de las asignaturas no } \\
\text { troncales y ofertadas por el Centro ¿qué } \\
\text { porcentaje representan las dedicadas a la } \\
\text { formación en IGOP? }\end{array}$ \\
\hline $\begin{array}{l}\text { Proporción de plazas cubiertas sobre las } \\
\text { ofertadas en las asignaturas de IGOP } \\
\text { optativas y de libre elección. }\end{array}$ & $\begin{array}{l}\text { Del total de plazas ofertadas en las } \\
\text { asignaturas optativas y/o de libre elección } \\
\text { ofertadas por el centro ¿qué porcentaje se } \\
\text { ha cubierto? }\end{array}$ \\
\hline $\begin{array}{l}\text { Proporción de alumnas matriculadas en } \\
\text { cada especialidad y curso }\end{array}$ & $\begin{array}{l}\text { Del total del alumnado matriculado en } \\
\text { cada especialidad y curso ¿qué tanto por } \\
\text { cien corresponde a mujeres? }\end{array}$ \\
\hline $\begin{array}{l}\text { Proporción de profesoras por especialidad } \\
\text { y curso }\end{array}$ & $\begin{array}{l}\text { ¿Qué porcentaje representan las mujeres } \\
\text { entre el total del personal docente? }\end{array}$ \\
\hline $\begin{array}{l}\text { Presencia femenina en cargos de } \\
\text { representación del profesorado y } \\
\text { directivos }\end{array}$ & $\begin{array}{l}\text { ¿Qué tanto por cien de cargos directivos } \\
\text { y de representación son ocupados por } \\
\text { mujeres? ¿Existe representación femenina } \\
\text { en todos los niveles? }\end{array}$ \\
\hline
\end{tabular}


Se trata de instrumentos de seguimiento que sirven para medir algunos de los aspectos de coeducación y que configuran las dimensiones de la escuela educativa (la intervención directa en el aula, los contenidos educativos, la formación del profesorado en igualdad, etc.), pero que se sitúan, igualmente, en el ámbito de actuación de los distintos agentes (dirección del centro, personal docente, instituciones para la igualdad, etc.). Las tablas agrupan los indicadores a partir de dos variables: 1) En función del agente que ha de contestar a las preguntas formuladas para dar respuesta a los indicadores planteados: profesorado, alumnado, profesorado y alumnado de forma conjunta y las autoridades educativas en distintos niveles; 2) En función de las distintas dimensiones de la educación a analizar, caso de la intervención directa profesorado-alumnado, los valores, los estereotipos sexistas reproducidos, la escuela como agente para el cambio y la formación del profesorado en Igualdad de Oportunidades (en adelante IGOP). A su vez, dentro de cada una de éstas, se diferencian los distintos ámbitos en los que se centra dicho análisis.

Uno de estos indicadores es la Proporción del tiempo asignado en materias troncales a la igualdad de Oportunidades (IGOP) y propone computar el total de créditos de asignaturas troncales por especialidad y curso para señalar qué porcentaje representan las consagradas a la formación en IGOP. Otro indicador es la Proporción de tiempo lectivo asignado a asignaturas sobre IGOP, entre las propias ofertadas por el centro, al margen de las troncales, es decir, del total de créditos de las asignaturas no troncales y ofertadas por el centro qué porcentaje representan las dedicadas a la formación en IGOP.

En el caso del Máster de Investigación en Didácticas Específicas, no se cumple con ninguno de estos indicadores ya que no aparecen asignaturas específicas dedicadas a la igualdad de género (tabla 2). Lo único que se detalla en las guías docentes es un apartado, dentro de las competencias a trabajar, en el que se indica la necesidad de «crear espacios de investigación y aprendizaje con especial atención a la equidad, la educación emocional y en valores, la igualdad de derechos y oportunidades entre hombres y mujeres, la formación ciudadana y el respeto de los derechos humanos que faciliten la vida en sociedad, la toma de decisiones y la construcción de un futuro sostenible.» Esta competencia también se recoge entre las competencias transversales especificas (CT2) incluidas para el conjunto del Máster (tabla 2), con 60 Créditos totales, 46 de carácter Obligatorio y 14 Optativos. 
Tabla 2. Desglose de asignaturas con créditos y carácter de las mismas

\begin{tabular}{|c|c|c|}
\hline Nombre & Créditos & Carácter \\
\hline Bases científicas & 6 & Obligatorio \\
\hline Bases didácticas 1 & 9 & Obligatorio \\
\hline Bases didácticas 2 & 7 & Obligatorio \\
\hline Bases para la innovación docente & 6 & Obligatorio \\
\hline Métodos de investigación didáctica & 6 & Obligatorio \\
\hline Trabajo fin de máster & 12 & Obligatorio \\
\hline Optatividad & 14 & Optativo \\
\hline $\begin{array}{l}\text { Investigación en didáctica de las Ciencias } \\
\text { Experimentales elementales }\end{array}$ & 7 & Optativo \\
\hline Investigación en didáctica de la Educación Física & 14 & Optativo \\
\hline Investigación en didáctica de las Artes Visuales & 14 & Optativo \\
\hline Investigación en didáctica de las CC. Sociales: geografía & 7 & Optativo \\
\hline Investigación en didáctica de las CC. Sociales: historia & 7 & Optativo \\
\hline $\begin{array}{l}\text { Investigación en didáctica de las Ciencias } \\
\text { Experimentales superiores }\end{array}$ & 7 & Optativo \\
\hline $\begin{array}{l}\text { Investigación en didáctica de las Matemáticas } \\
\text { elementales }\end{array}$ & 7 & Optativo \\
\hline $\begin{array}{l}\text { Investigación en didáctica de las Matemáticas } \\
\text { superiores }\end{array}$ & 7 & Optativo \\
\hline Investigación en Educación lingüística & 3,5 & Optativo \\
\hline Investigación en Educación literaria & 3,5 & Optativo \\
\hline Investigación en Educación musical & 14 & Optativo \\
\hline $\begin{array}{l}\text { Investigación fundamental en didáctica de las Ciencias } \\
\text { Experimentales }\end{array}$ & 7 & Optativo \\
\hline $\begin{array}{l}\text { Investigación fundamental en didáctica de las } \\
\text { Matemáticas }\end{array}$ & 7 & Optativo \\
\hline Metodología de la Educación lingüística & 3,5 & Optativo \\
\hline Metodología de la Educación literaria & 3,5 & Optativo \\
\hline
\end{tabular}

Otro indicador propuesto es la proporción de alumnas matriculadas en cada especialidad y curso del total del alumnado matriculado en cada especialidad y curso, que trata de determinar qué porcentaje corresponde a mujeres. En el caso del máster que nos ocupa, de las 92 personas inscritas este curso el $57^{\circ} 6 \%$ son mujeres. El siguiente indicador que se propone recoger es la proporción 
de profesoras por especialidad y curso, midiendo qué porcentaje representan las mujeres entre el total del personal docente, así como la presencia femenina en cargos de representación del profesorado y directivos, que trata de establecer qué tanto por cien de cargos directivos y de representación son ocupados por mujeres y si existe representación femenina en todos los niveles. En este sentido, las profesoras representan el $31 \%$ del total de personal docente, el mismo porcentaje que en cargos directivos, aunque consideramos especialmente destacable que sea una mujer quien dirija el Máster (tabla 3).

Tabla 3. Número y porcentaje de representatividad por sexos en el personal docente y directivo en el Máster de Didácticas Específicas de la Universitat de València

\begin{tabular}{|l|r|r|r|r|c|}
\hline \multicolumn{1}{|c|}{ Nivel } & \multicolumn{2}{|c|}{ Hombres } & \multicolumn{2}{c|}{ Mujeres } & Totales \\
\hline & $\mathrm{n}$ & $\%$ & $\mathrm{n}$ & $\%$ & \\
\hline Dirección & 0 & & 1 & & 1 \\
\hline Comisión Académica del Máster & 5 & & 2 & & 7 \\
\hline Secretaría & 1 & & 0 & & 1 \\
\hline Coordinadores de Especialidad & 5 & & 2 & & 7 \\
\hline Total Puestos directivos & 11 & $69 \%$ & 5 & $31 \%$ & $16(100 \%)$ \\
\hline Profesorado Educación Física & 4 & & 1 & & \\
\hline Profesorado Lengua y Literatura & 6 & & 8 & & \\
\hline Profesorado música & 4 & & 1 & & \\
\hline Artes visuales & 2 & & 1 & & \\
\hline Profesorado Ciencias Experimentales & 10 & & 4 & & \\
\hline Ciencias Sociales & 6 & & 0 & & \\
\hline Profesorado Matemáticas & 5 & & 2 & & \\
\hline & 37 & $69 \%$ & 17 & $31 \%$ & \\
\hline Total docentes & 48 & $69 \%$ & 22 & $31 \%$ & $70(100 \%)$ \\
\hline
\end{tabular}

En el análisis total de la representación femenina en el Máster (gráfico 1), se aprecia una clara preponderancia del género masculino sobre el femenino en todos los ámbitos del mismo, salvo entre el profesorado de Lengua y Literatura, donde hay dos mujeres más que hombres dentro del equipo docente. 


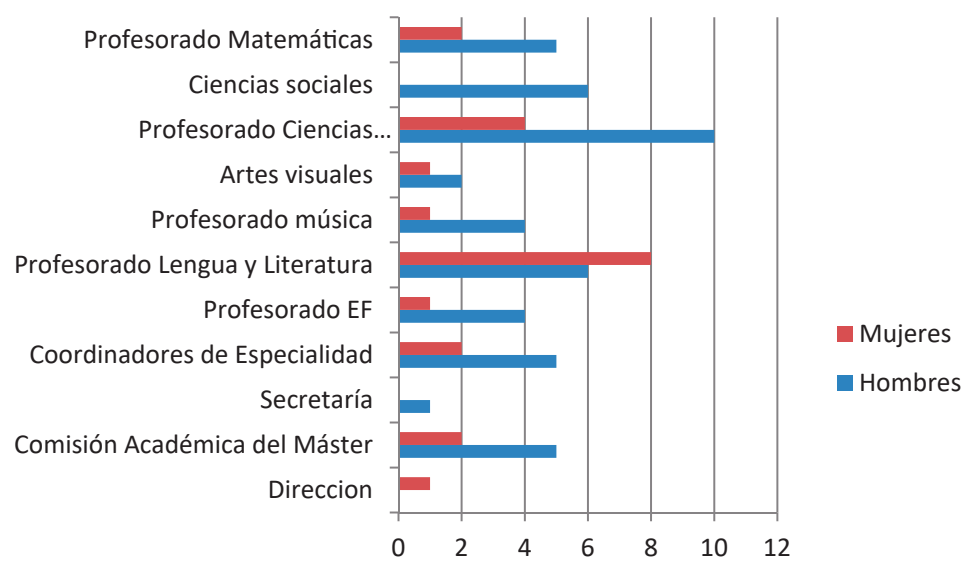

Figura 1. Representación total de las mujeres en el Máster de Investigación

\subsection{Materiales y actividades propuestas}

Los materiales son fundamentales en el proceso de transmisión cultural, porque concretan la selección con la que vamos a trabajar, la que vamos a presentar a los y las estudiantes (Blanco 12). En este sentido, consideramos que una selección pensada de los mismos puede ser determinante para alcanzar los objetivos deseados. También para crear un contexto de enseñanza-aprendizaje que intente potenciar en el aula "procesos cognitivos más ricos que los que se dan en una simple situación de transmisión oral cuya finalidad sería su posterior restitución por parte de los alumnos» (Dalongeville 5), para conseguir un aprendizaje más significativo o para contribuir a un trabajo competencial.

Por ello, la propuesta de trabajo que se plantea para incorporar el género dentro el Máster de Investigación de Didácticas Específicas estaría concebida a partir del desarrollo de los siguientes apartados:

1. Una primera aproximación que permita evidenciar y hacer patentes las representaciones sociales de nuestro alumnado a través de los estereotipos de masculino y femenino. La razón es que «los alumnos aprenden por reestructuraciones sucesivas que integran lo sabido y lo nuevo, lo reinterpretan y modifican su sentido» (Benejam 76). Por ello, no puede haber un aprendizaje significativo en el que las representaciones no emerjan en un primer momento y en las que éstas no sean puestas en 
tela de juicio, para que no se conviertan en un obstáculo que impida que la construcción de nuevos conocimientos (Dalongeville 5 y 8 ).

2. Un sondeo a los conocimientos previos de nuestro alumnado, en relación con el papel de las mujeres en las Ciencias Experimentales y Sociales.

3. Una reflexión acerca de los roles y estereotipos de género, entendidos como el conjunto de normas sociales y comportamentales generalmente percibidas como apropiadas para los hombres y las mujeres en un grupo o sistema social dado, en función de la construcción social que se tiene de la masculinidad y femineidad.

4. Un análisis de una selección de textos, de diferentes autores clave en la cultura occidental, en la que éstos reflexionan sobre la condición y el papel social de las mujeres. Estos dos últimos pasos adaptarían algunas actividades propuestas por la guía didáctica Mujeres en la Historia publicada por el Instituto Asturiano de la Mujer (2010).

Querríamos comenzar realizando un trabajo previo sobre las representaciones sociales de nuestro alumnado desde la perspectiva de género, cuestionándoles sobre aquello que, en su opinión, el entorno social actual demanda a los hombres y a las mujeres. El objetivo es positivar y/o hacer evidentes las demandas a las que nos someten nuestras sociedades y sistemas culturales en función de las identidades de género, pero también, por qué no, tratar de subvertir los roles. Se concibe como una actividad planteada para cuestionar los estereotipos, entendidos como imagen o idea aceptada comúnmente por un grupo o sociedad con carácter inmutable.

A continuación, se planteará un ejercicio que evidencie la invisibilidad de las mujeres para la historia y las Ciencias Experimentales y Sociales. Se trata de una actividad en la que pediríamos al alumnado que escribiera el nombre de veinte hombres y veinte mujeres que considerasen importantes en el transcurso de la historia, la geografía y/o la historia del arte y el nombre de veinte mujeres científicas. Trataríamos con ello de revisar el concepto tradicional de «protagonista» de la historia y también el de científico y/o científica vinculados aún hoy en día a una noción androcéntrica y tradicional (Fernández Valencia 61).

Otro de los ejercicios presentaría, en forma de tabla o esquema, los roles o funciones sociales tradicionalmente asignados a los diferentes géneros en las sociedades patriarcales, para ver hasta qué punto nuestros alumnos y alumnas se identifican con ellos o consideran que siguen estando vigentes en las sociedades actuales. Los primeros asociarían a la mujer con el espacio privado, la invisibilidad, la pasividad, la naturaleza, la emotividad, la debilidad física y 
la inteligencia emocional. Los segundos la contrapondrían a un concepto de hombre y de masculino vinculado a la esfera pública, la visibilidad, la actividad, la cultura y la creación artística, la razón, la fuerza física y la inteligencia racional.

Por último, quisiéramos proponer el análisis de una selección de textos breves, suscritos, como señalábamos, por diferentes autores clave en la cultura occidental (VVAA 2010). Entre ellos se cuentan autores clásicos como Aristóteles, Pitágoras o Platón, pero también ilustrados célebres (Rousseau), filósofos contemporáneos (Nietzsche) o médicos (Gregorio Marañón). Igualmente, fragmentos extraídos del Nuevo Testamento o suscritos por reformadores protestantes como Lutero. Su incorporación responde al hecho de que consideramos el trabajo con fuentes como una herramienta esencial en clase, pero también a que los discursos son uno de las prácticas fundamentales para construir la diferencia de género. Por ello consideramos que es necesario conocerlos, para poder decodificarlos y ser capaces de adoptar, con posterioridad, una postura crítica frente a los mismos.

En esta selección de textos, autores considerados canónicos y destacados como referentes dentro de diferentes disciplinas presentan, literalmente, a la mujer como inferior al hombre y a éste como superior y como «director» frente a ella (Aristóteles). También se las vincula con un principio «malo», creador del caos, la oscuridad y la mujer (Pitágoras), o como reencarnación en una segunda vida de aquellos hombres que fueron cobardes en la primera (Platón). Igualmente, se reclama su silencio y su sumisión (Nuevo Testamento), se la compara con la «mala hierba» (Lutero), se le exige dulzura y sometimiento al «tratamiento injusto» del marido (Rousseau), sumisión sexual (Nietzsche) o aceptación incondicional de la maternidad como característica básica de su sexo (Marañón). Se trata, en suma, de ofrecer al alumnado algunos de los textos que articulan el discurso de la misoginia, entendida como aversión a las mujeres y que, a lo largo de la historia, han configurado la visión de «la mujer» en las sociedades occidentales, utilizando la biología, la religión, la filosofía, la ciencia, etc., para argumentar su inferioridad.

\section{CONCLUSIÓN}

Del análisis presentado se puede deducir que, en el caso del Máster de Investigación en Didácticas Específicas de la Universitat de València, la dedicación académica al tema de la coeducación, así como la presencia equitativa de ambos sexos en la composición docente y directiva del Máster no es considerada como prioritaria, ya que a lo largo del periodo formativo no hay una 
atención exclusiva al tema de igualdad entre hombres y mujeres ni se cumplen las relaciones equitativas de género. Creemos que, tratándose de un máster que posee una doble vertiente de formación y de investigación, la falta de formación con perspectiva de género va a dificultar, a la larga, el poder dotar al alumnado de un marco de interpretación de la realidad que sea capaz de encajar con mayores elementos de justicia social. Además, ha quedado expuesto y avalado por la bibliografía existente (Donoso-Vázquez 76) que la formación en género en la formación superior se impone por varios motivos, entre los más importantes, que construye un mundo más equitativo y humano. Por lo que respecta a la vertiente investigativa, el no dotar al alumnado de esta perspectiva, la que supone la introducción de una categoría de análisis como género, dificulta que éstos puedan ampliar sus concepciones, abrir nuevas líneas de investigación y, sobre todo, cuestionar y revisar algunas de las conclusiones establecidas desde una posición androcéntrica.

Nuestra propuesta de actividades está por tanto dirigida a trabajar la coeducación en los procesos de aprendizaje y a incentivar una investigación con perspectiva de género, con la finalidad de que los avances en la construcción social del conocimiento sean difundidos y conocidos por el conjunto de la sociedad, porque a ella pertenecen. De lo contrario, éstos permanecen como un bagaje de conocimiento exclusivo y cerrado de la comunidad científica de las diferentes disciplinas. Puesto que la educación posee, entre otras, la función social de transmitir el conocimiento y la cultura científica a los más jóvenes a través de la educación formal, creemos que es necesario que esta educación, inicial o especializada, incorpore el género y sus derivaciones en todas las propuestas y ámbitos.

Sólo con una experiencia de formación en perspectiva de género se pueden conseguir cambios en los procesos mentales que se dan en el alumnado que contribuyan a modificar sus representaciones sociales. Los estudiantes debe asimilar y hacer suyos los conceptos asociados al género, además de ser capaz de generar respuestas genuinas, críticas y activas propias. De conseguirlo, los cambios sugeridos implicarían el desarrollo de una conciencia de la perspectiva de género, un análisis crítico de las realidades contextualizadas y una motivación que podría llegar a ser determinante para impulsar aquellos cambios que creemos son tan necesarios en nuestra sociedad. 


\section{REFERENCIAS BIBLIOGRÁFICAS}

Amelang, James y Mary Nash (comps.). Historia y género. Las mujeres en la Europa moderna y contemporánea. Valencia: IVEI, 1990.

Benejam, Pilar. «La selección y secuenciación de los contenidos sociales». Enseñar y aprender Ciencias Sociales, Geografía e Historia en la educación secundaria. Benejam, Pilar y Joan Pagès (coord.). Barcelona: Institut de Ciències de la Educació, (1997): 71-95.

Blanco García, Nieves. «Coeducación: la apuesta por una pedagogía de sujetos visibles». Kikiriki. Cooperación Educativa 54 (1999): 19-20.

Blanco García, Nieves. «Los saberes de las mujeres y la transmisión cultural en los materiales curriculares». Investigación en la escuela 65 (2008): 11-22.

Conselleria de Educación, Investigación, Cultura y Deporte. «Instrucción del 15 de diciembre de 2016, del Director General de Política Educativa, por la que se establece el protocolo de acompañamiento para garantizar el derecho a la identidad de género, la expresión de género y la intersexualidad». [2016/10425] del 27 del 12 de 2016.

Dalongeville, Alain. «Noción y práctica de la situación-problema en historia» Enseñanza de las Ciencias Sociales 2 (2003): 3-12.

Donoso-Vázquez, Trinidad y Anna Velasco-Martínez. «¿Por qué una propuesta de formación en perspectiva de género en el ámbito universitario?» Profesorado. Revista de Curriculum y Formación del Profesorado 17. 1 (2013): 71-88.

Elizondo, Arantxa, Ainoa Novo y María Silvestre. «Igualdad de mujeres y hombres en las universidades españolas». Madrid: Instituto de la mujer. Ministerio de Sanidad, Política Social e Igualdad, 2010.

Fernández Valencia, Antonia. Las mujeres en la enseñanza de las Ciencias Sociales. Madrid: Síntesis, 2001.

Heredero De Pedro, Carmen y Esther Muñoz Hernández. «Más y mejor coeducación». Experiencias. CEE Participación educativa 11 (2009): 139-145.

Hidalgo, Encarna, Dolores Juliano, Monserrat Roset y Àngels Caba. Repensar la enseñanza de la geografía y la historia. Una mirada desde el género. Barcelona: Octaedro, 2003.

Jaramillo Guijarro, Concepción. «Formación del profesorado: igualdad de oportunidades entre chicas y chicos». Madrid: Ministerio de Trabajo y Asuntos Sociales. Instituto de la mujer, 1999.

Menéndez, Cristobal. «El papel de la mujer en la investigación científica y médica en el siglo XXI: un debate necesario». Aten Primaria 43 (2011): 331-2.

Pagès, Joan. «La didáctica de las Ciencias Sociales y sus retos». Vallés, Joan, Álvares, Dolores y Rickennmann, Réné (eds.). La activitat docent. Intervenció, innovació, investigació. Gerona: Documenta universitaria, 2011, 45-62. 
Rausell Guillot, Helena. El papel de las mujeres en la sociedad actual. Madrid: Santillana, 2016.

Rebollar Sánchez, Edith Mariana. El género en los planes de estudio de los grados de educación de las universidades públicas españolas. Tesis doctoral. Universidad Autónoma de Barcelona: Barcelona, 2013.

Red2 (red de consultores s.l.). Guía de coeducación. Documento de síntesis sobre la educación para la igualdad de oportunidades entre mujeres y hombres. Madrid: Instituto de la Mujer. Observatorio para la igualdad de oportunidades, 2007.

Romero, Alfons y Paco Abril. «Género y formación del profesorado en los estudios de educación infantil». REIFOP 11.3 (2008): 43-51. Disponible en: <http:// www.aufop.com/> Consultado el 19-05-2017.

Valls, Rafael. «La exaltación patriótica como finalidad fundamental de la historia en la educación obligatoria: una aproximación histórica». Didáctica de las Ciencias Experimentales y Sociales 5 (1991): 33-47.

VVAA. Mujeres en la Historia. Guía didáctica. Oviedo: Instituto Asturiano de la Mujer, 2010. 